\title{
Why we need more debate on whether psychotic symptoms lie on a continuum with normality
}

\author{
A. S. David* \\ Section of Cognitive Neuropsychiatry, Institute of Psychiatry, King's College, London, UK
}

The notion that psychotic symptoms lie on a continuum with normality has become an accepted dogma. It is supported by several lines of empirical evidence, fits in with the orientation of modern services and has a 'moral' appeal. However, there is confusion as to the nature of the continuum or continua under discussion. According to the author, commentators on this topic do not often distinguish between the variability and severity of the phenomena themselves, within or between individuals, versus the distribution of symptoms or risk factors in a population. The implications of these two types of continua differ. Furthermore, the evidence for continua of delusional beliefs and hallucinations can be challenged on a number of grounds, both methodological and conceptual. To some extent, whether phenomena are viewed as continua or categories depends on the intentions of the observer. Finding the distinctive characteristics of psychotic phenomena in people with clinical disorders, in addition to their origins in 'normal' cognitive processes, is a worthwhile goal.

Received 28 July 2009; Revised 5 January 2010; Accepted 11 January 2010; First published online 11 February 2010

Key words: Continuum, delusions, hallucinations, psychosis, schizophrenia.

\section{Introduction}

The notion that apparently healthy people can experience psychotic symptoms such as delusions and hallucinations - the continuum concept or theory of psychosis - has been explored for over 100 years. Since around the mid-1980s it has gained a large number of adherents so that it is now becoming the accepted dogma. Although the theory can call upon a relatively large body of evidence in its support, drawn from phenomenological studies, epidemiology, developmental psychopathology and cognitive psychology (Johns \& van Os, 2001; Freeman, 2007; Stip \& Letourneau, 2009; van Os et al. 2009), there is also work that suggests that viewing all psychotic phenomena as continua may lead us down a blind alley in understanding the nature of psychosis.

Before tackling these questions it is important to define the area of discourse. Previous category versus continuum debates were sometimes rather crude affairs, with the proponents on the category side seeing themselves as the defenders of psychiatry against 'mental illness deniers', or the debate was seen, inappropriately, as a biology versus humanities contest. I wish to distance myself from simplistic polarization

* Address for correspondence: A. S. David, M.D., FRCP, FRCPsych., M.Sc., Section of Cognitive Neuropsychiatry, PO Box 68, Institute of Psychiatry, King's College, Denmark Hill, London SE5 8AF, UK.

(Email: anthony.david@kcl.ac.uk) of this debate. Having said that, identifying possible parallels between the biomedical and psychosocial sciences and the way each treats disease concepts may be of value in reviving support for the idea of categories of mental phenomena and providing a sound basis from which to critique the continuum theory.

Ernst Kretschmer was the first to clearly articulate the continuum idea when he stated: 'endogenous psychoses are nothing other than marked accentuations of normal types of temperament' (Kretschmer, 1925). However, Paul Meehl, the American psychometrician, is most often credited with providing the modern intellectual bedrock for the continuum theory (Meehl, 1962). Meehl was discussing what we would now call endophenotypes, or biomarkers, with respect to schizophrenia. He was prompted to examine the issue because of accumulating evidence that people with a strong family history of schizophrenia exhibit certain traits that fall short of the schizophrenia syndrome yet nevertheless seem abnormal or dysfunctional in some way ('schizotaxia'). What he really sought was a 'hard' biological equivalent of what were then and remain somewhat vague and difficult to define personality characteristics. He concludes: 'It is my strong personal conviction that such a research strategy will enable psychologists to make a unique contribution in the near future, using psychological techniques to establish that schizophrenia, while its content is learned, is fundamentally a neurological disease of 
genetic origin.' In other words, his aim was to deploy cognitive and behavioural continua to help define the disease category called schizophrenia, not to dispense with the category altogether.

\section{Strengths of the continuum view of psychosis}

The first strength to the continuum view is that it reflects uncertainties in clinical practice, where, clearly, abnormality shades with normality, where the deluded overlap with the eccentric, and where, among other things, schizophrenia blends with affective disorder and personality subtypes. Next, as noted above, it encourages theory development within models of normal cognition so that symptoms are not so 'ununderstandable' in the Jasperian sense but can be seen as distortions or exaggerations of common phenomena. Contrary to some polemicists (Read \& Harre, 2001), this is entirely consistent with what might be called the 'medical model'; for example, the symptoms and signs of any organ failure (kidney, liver, heart) can only be understood from a model of normal organ functioning. As I have argued elsewhere (David, 1993), this is the essence of the cognitive neuropsychiatric approach and should be a two-way process. Healthy functions may sometimes be inferred only when lost through disease or dysfunction.

Another strength of the continuum view is that it facilitates exploration of quantitative aetiological factors such as multiple genes of small effect; 'stress', etc. This lies behind much current research searching for intermediate or endophenotypes. A practical benefit is that the continuum concept justifies the study of healthy subjects who lie at some point on it, who may be more accessible than patients and certainly free of many potential confounds to research, particularly medication and other secondary effects of illness. Finally, although the continuum account of psychosis will stand or fall on the basis of empirical evidence, such accounts can take hold and endure in the minds of researchers and lay people alike because they seem concordant with a larger world view (see Kessler, 2002). Hence, a strong motivation for adhering to the continuum account of psychosis is the moral benefits of this position. It is the notion that 'we are all a bit mad' that is thought bound to reduce stigma and help break down any alienating sense of 'us and them' (Bentall, 2003).

The current revival of the continuum view has flowed from evidence from modern molecular genetic and developmental research, which supports an extended genetic phenotype of psychosis. The most powerful recent strand comes from longitudinal studies, such as the Dunedin cohort study (Poulton et al. 2000), which examined children from
New Zealand from childhood to adulthood and found that teenage children frequently endorsed symptoms akin to psychotic hallucinations and abnormal beliefs. What elevated this finding from a curiosity was the fact that such children grew up to have a higher risk of schizophreniform disorder. Alongside this, clinicians have become interested in the prodrome of schizophrenia. People said to be in the midst of the prodrome are apparently found to exhibit variously defined versions of psychotic symptoms either with attenuated phenomenology or instead with full-blown symptoms but occurring only transiently (Yung et al. 2003).

As for surveys of psychotic symptoms in the general population, studies of increasing sophistication and epidemiological rigour are being published and have been comprehensively reviewed (Stip \& Letourneau, 2009; van Os et al. 2009). They find that surprisingly high proportions of seemingly healthy adults or young people, whether they be random samples of the population or primary care attenders, endorse symptoms akin to hallucinations and delusions.

\section{Weaknesses in the evidence}

There are two important observations to make about all these studies. First, it soon becomes evident after only a cursory perusal of the literature that even workers motivated by the idea of a continuum find it impossible to resist setting a threshold above which a hallucination or delusion may be defined. Hence, the phenomena are described as possible or probable or definite, or a distinction is made between items endorsed from a questionnaire and those elicited by lay interviewers or through clinical interview and subsequently verified (Laurens et al. 2007; Horwood et al. 2008). This methodology seems perfectly apt. However, the obvious finding is that it matters a great deal how the phenomena are elicited, checked and verified as to just how large the proportion of those experiencing the phenomena will turn out to be. The ranges quoted 'vary substantially' (van Os et al. 2009), and many quasi-psychotic phenomena turn out to occur in the majority of children and adolescents before they are boiled down to the $8 \%$ with psychotic experiences and thence the $3 \%$ with psychotic disorder, about a third of which persist.

Hence, rather mundane methodological issues can exert a large effect on whether one is led to conclude that psychosis-like symptoms are common or rare in the population in question. Another methodological issue concerns the nature of the questions and the expectations they engender in participants. The Dunedin questionnaire has served as a template for many 
similar studies (Poulton et al. 2000; Horwood et al. 2008), and used the schizophrenia section of the Diagnostic Interview Schedule for Children to elicit phenomena. Questions begin with a preamble such as: 'Some people believe in mind reading or being psychic. Have other people ever read your mind?' This type of leading question might be justified in a clinical situation to put a frightened and distressed young person presenting with such experiences at ease. However, there is a possibility that it will encourage false positives in non-clinical groups because they are not $a$ priori expected to have such symptoms. The effect of such framing in surveys of different populations would be a worthwhile topic for methodological research. Even if biased questioning affected rates of symptoms in all types of population, work of this kind tends to emphasize the high prevalence on the nonclinical group rather than the (also) high relative risk for such symptoms in those clinically identified (e.g. Verdoux et al. 1998).

\section{Two kinds of continua}

\section{Epidemiology}

Continua can be considered on two levels: the population level and the phenomenological level. Foremost in those leading epidemiological studies is psychiatrist Jim van Os and colleagues, who have exploited numerous cohort studies and applied to them sophisticated statistical analytic techniques (van Os et al. 2009). However, their epidemiological approach can be contrasted with the phenomenological approach. Consider a population in which each member may possess a trait that, when plotted, might assume a normal distribution. This might be called a type 1 continuum. This can be contrasted with a continuum of phenomena that can occur within an individual who may be healthy or may have been diagnosed as suffering from psychotic disorder; this is close to what British psychologist Gordon Claridge called a quasicontinuum (Claridge, 1994). Thus, various forms of hallucination-like experiences may be thought to exist on a continuum that again may be normally distributed or more likely distributed along a half-normal distribution, that is with most people not experiencing the phenomenon at all and with a small tail experiencing the phenomenon in its most extreme form. This might be called the type II continuum. It should be said that Bentall (2003) notes the two types of continua but goes no further because, for his purposes, they each support the notion that there is no clear dividing line between the sane and the insane. This illustrates the moral imperative to the category versus continuum debate.
The importance of the epidemiologically based continuum view can be traced back to the work of epidemiologists such as Geoffrey Rose in England (Rose, 1992; cited approvingly by Verdoux et al. 1998 and Verdoux \& van Os, 2002) and is twofold: first, it provides a rationale for prevention or early intervention, and second, it is potentially refutable (see later). On the first point Rose made the observation that small changes affecting a large number or even the majority of a population can have much greater health benefits than those that target members of the population at the extreme end of a distribution. So, classically, blood pressure might be normally distributed and there is a continuum of risk: the higher the blood pressure the greater the risk of stroke and heart disease. However, Rose's approach would be to favour an intervention that generally lowered the population blood pressure by a small amount, thus shifting the entire distribution leftwards so that there were fewer crossing a threshold of pathological consequence. An equivalent in psychiatry might be the relationship between low mood and suicide. An intervention, whatever that might be, aimed at improving mood by a small amount in an entire population would theoretically have a greater impact on suicide compared to an intervention aimed only at those with severe depression, while accepting that the latter group has a particularly high suicide risk. The idea here is that even those with relatively mild symptoms of depression are at a somewhat increased risk of suicide, and given that there are so many more people with mild depression than severe depression, a slight change in the former group will have a greater cumulative impact on suicide rate. However, viewing risk as a continuum is very different from using the notion of a continuum to undermine nosological categories, as occurs in the psychiatric literature. In the above examples, suicide and stroke are clearly categorical outcomes that are not in any way undermined by being related to a continuum of risk. To date, no-one has extended the work on the continuum of psychosis to produce a population-level intervention (although such ideas have been mooted; Mojtabai et al. 2003). One example would be putting antipsychotic drugs in the water supply or educating children against jumping to conclusions, thereby reducing the propensity to psychosis in the population, but these are unlikely to find general support (see Charlton, 1995; McGorry, 2005).

\section{Phenomenology}

In an article used to relaunch the idea of a continuum (van Os et al. 2000), the authors pay homage to the classic paper by John Strauss written in 1969 (Strauss, 
1969). Strauss was talking about the type II or phenomenological continuum. It is worth revisiting his paper in detail. This was a study carried out as part of an international effort to improve the diagnosis of schizophrenia. Using the Present State Examination (PSE), Strauss focused on those patients whose ratings of delusions could not be scored confidently by the raters. Note that he was not looking at those who scored midway between zero and full-blown delusions, which would have been easy within the PSE 0-1-2 scoring system. Instead he isolated several phenomena that were somewhat unstable, oscillating between true delusions and delusion-like phenomena, or fixed impervious delusions that were suddenly questionable in the eyes of the sufferer. Strauss concluded that the delusions themselves formed a continuum and that this perhaps had something to do with the recovery process (Stanton \& David, 2000).

The other recent group of studies to contribute evidence to the debate are those examining hallucinations in social groups who identify themselves as voice hearers. There are now several such studies, facilitated by the internet, that make a convincing case for there being otherwise healthy individuals who have, to all intents and purposes, psychotic auditory hallucinations. These people do not seem to have a progressive disorder and certainly do not seem to be disabled by their symptoms, unlike 'regular' patients. Such individuals cast doubt on diagnostic categories by suggesting a continuum of disability. One of the largest collections of such people has been gathered by Iris Sommer in The Netherlands (Sommer et al. 2008). She has laid out the phenomenology of this group in comparison to other healthy controls and patients with schizophrenia to highlight the similarities and differences (Daalman et al., in press). This important work can be seen as providing ammunition for parties on both sides of the continuum-category debate. Certain aspects of auditory hallucinations traditionally viewed as pathological, such as the location of the voice inside or outside of the head, seem to have almost no value in distinguishing so-called pathological and benign hallucinations. Similarly, the subjective reality of the hallucinated voices was identical in both groups. However, when looked at more closely, there are characteristics of the voice, not the inside/outside distinction but the emotional content of the voices, frequency and age of onset, and the fact that some 'voices' seem to have a specific focal location, that are exclusive to patients with schizophrenia. Using the patient versus non-patient distinction, it is possible to calculate the odds ratios (ORs) of certain phenomena occurring (categorically) in one group and not the other and these may be very high and the $95 \%$ confidence intervals (CIs) clearly excluding unity. For example, 82/111 (74\%) non-patient auditory verbal hallucinators versus $57 / 118(48 \%)$ psychotic patients gave a predominantly external attribution to their voices (OR 3.03, 95\% CI 1.73-5.28). Thirty patients, but not one of the non-patients, attributed them to specific people or things, for example devices implanted in the brain (5) or other (living) people (19) (OR $\approx 90.1,95 \%$ CI 11.7-689).

Another important characteristic of schizophrenic hallucinations is their hostile and distressing nature. Although benign encouraging hallucinations do occur in schizophrenia as in non-pathological conditions, it seems that only the critical and intrusive voices, those liable to cause distress, never occur in healthy 'voice hearers' (Nayani \& David, 1996; Sommer et al. 2008; Daalman et al. in press). Looking at the question from the other perspective, how similar are the so-called 'subclinical' phenomena to traditional clinical phenomena? In a recent study, Simon et al. (2009) helpfully describe in detail the content of subclinical hallucinations in 84 adolescent out-patients. In the auditory domain, that most relevant to psychosis, the categories and numbers of cases associated with them were: voices calling their own name (nine), acoasms (i.e. noises) (six), altered perception of auditory stimuli (six), hearing music (four), unrecognizable murmuring voices (three), steps (two), school bells or cell phone ringing (two), echoes (one), hearing own thoughts as a voice in the head (one), and whispers (one). Clearly, none of these would be mistaken for a psychotic symptom by a trained clinician.

The importance of distress is also emphasized by Emmanuelle Peters and colleagues when considering delusions and delusional-like ideas on a continuum. Peters et al. $(1999,2004)$ devised a scale specifically to tap into these phenomena. In so doing they naturally altered the description of the phenomena so that they would be acceptable for the general population. Although this shows that such beliefs and ideas occur in the population, it would be wrong to conclude that such people were on the same continuum as those with true delusions. Peters et al. added the expression 'as if' when describing the delusions and used only normal or conventionally spiritual examples: for example, 'Do you ever feel as if people are reading your mind ?' and 'Do you feel as if things in magazines or on TV were written especially for you?' (emphasis added).

Putting aside the fact that the phenomenology cannot be claimed to be delusional whatever findings emerged, it therefore seems that patients clinically designated as being deluded share many of the same beliefs in terms of content as healthy people. Indeed, the number of odd beliefs held does not distinguish the groups very satisfactorily either (but see Lincoln, 
2007). However, the groups do differ markedly on several other dimensions (see also Verdoux et al. 1998). Peters and colleagues emphasize that distress is something that marks out the clinical group, but this group also scores significantly more highly than control groups on preoccupation and conviction. It should also be noted that this study and many like it compare treated patients on medication with controls, which will naturally tend to bring the patient group nearer to the comparison group on any given measure of pathology, producing more overlap and the appearance of a continuum.

Work by Peters and colleagues has tended to concentrate most on distress and explanations for it in asking the question: what makes certain beliefs pathological? However, they might equally consider conviction, that is the strength of the belief, because this is a more traditional measure of what distinguishes delusions from other beliefs. Tania Lincoln has used the Peters Delusion Inventory (PDI) on deluded people and other populations (Lincoln, 2007). In many ways she replicates Peters et al.'s original findings but is just as interested in those factors that mark out the clinical group as the overlap between them and the comparison group(s). Lincoln has kindly provided me with correlation matrices from this and a subsequent survey (Lincoln \& Keller, 2008). The results showed that distress and conviction correlate fairly strongly in the deluded group $(r=0.479, n=54)$ and more so than in a healthy volunteer group $(r=0.392, n=359)$, suggesting that the traditional, definitional property of a delusion, namely conviction, is indeed related to distress, although of course a simple correlation cannot determine the direction of causality. Preoccupation was most strongly related to distress ( $r=0.646$ in patients and $r=0.751$ in controls).

In clinical experience it does seem to me that certain beliefs, if held with conviction, could never be anything but preoccupying and distressing, and that other factors such as esteem, emotional, regulation, previous stress and lack of social support need not be invoked to explain why beliefs become pathological (cf. Freeman, 2007). For example, I once attempted to look after a patient who believed that the hospital she was in was likely to be taken over by a large supermarket chain (true) and that the patients would be used to provide meat for sale (untrue). The belief included the fear that she would be strapped down upon a meat slicing machine to provide 'ham' for the delicatessen counter. It is impossible to imagine holding this belief with even the slightest degree of conviction and not being distressed by it. The content is all; how it came about remains a mystery.

Lincoln's work using the PDI can be used to illustrate this (Lincoln, 2007). Conviction, preoccupation and distress are tied closely together, especially in patients in whom all scores are generally higher. Where conviction, preoccupation and distress dissociate is where the beliefs have an obvious content (and context) and where they shade into spiritual (mostly positive) or religious beliefs (e.g. that one is chosen by God), a well-known pitfall for the unwary phenomenologist seeking to categorize delusions separately from other irrational beliefs (David, 1999). Where the dimensions of belief come together (i.e. covary most strongly) is when the content is that of Kurt Schneider's first-rank symptoms.

\section{Psychological versus biological continua}

It is perfectly valid to place biological or physical measures on a continuum (blood pressure, height, daylight, etc.) but we need to consider whether this applies to psychological phenomena. Of course it is quite simple to create a continuous measure for something like mood with an analogue scale or by adding up items on a questionnaire. Given the need to encompass at least some familiar phenomena in the scale and the inevitable error of measurement surrounding items on such a scale, we are bound to find scores on a distribution, be it normal, skewed or conforming to some other pattern. However, the content of such scales lacks any external validation. This is more problematic for psychosis, which intuitively lacks a single obvious dimension and, as work by Peters, Lincoln and others has shown, is best represented by several dimensions that may intercorrelate surprisingly weakly (Garety \& Hemsley, 1987). Furthermore, concepts such as 'conviction' or 'preoccupation' are complex phenomena in their own right, and could be divided meaningfully into several subdimensions. Delusions may be measured on more than 10 dimensions, including conviction, preoccupation, distress, absurdity, self-evidentness, pervasiveness; more than three or four dimensions are difficult to conceptualize. Instead, we are encouraged to squeeze this genuine complexity into a number on an inventory and then wonder at how variable the scores are in a population. This is not an argument against quantifying phenomena and subsequently comparing groups on how they score but it raises questions about how to interpret the results.

The difficulty with psychopathological continua is that we really do not understand which particular aspects or dimensions are important and how to weigh one against another. As an analogy, consider histopathology and the continuum of normal and abnormal tissue growth. At some point physicians have to determine what is benign and what is malignant. Consider, for example, the size of lesion, its microscopic 
appearance, its propensity to metastasize, to bleed, to cause pain, and so on. It is easy to add up scores on a scale formed by these and similar items and discuss malignancy as a continuum and compare cancer cases with healthy controls or those with benign lesions and show the overlap. However, such a dimensional measure may not be meaningful. For example, is a large lesion 'worse' than a small one? Indeed, very large tumours may be benign, so including this measure could distort the continuous scale. So a high score on this scale, made up of a ragbag of measures, all with equivalent face validity, would be a very poor guide to whether the growth in question was cancerous. In the case of physical disease we need not resort to such inherently weak methods because our knowledge of pathology enables us to pick out the features that really are decisive and meaningful; the propensity to metastasize being an obvious one. Without such knowledge, as in the mental health field, we have to resort to scales with little theoretical basis, which might be positively misleading. Hence, 'number of voices' and their situation inside or outside the head, etc., continue to add their number to scores on a supposed continuum of hallucinations that may conceal truly pertinent features (if only we knew which) and whose anchor points are unknown. Mullen (2003) has argued that we are mistaken to think of delusions as simple entities with limited dimensions of equal importance. They are, instead, multiply determined and can only be considered in their social and experiential context.

There are more complex statistics that may be able to help us here, to separate people or phenomena that differ along multiple dimensions, such as latent class analysis (Everitt et al. 2001). Indeed, I am grateful to Professor van Os for sharing with me a recent paper (Linscott \& van Os, 2010) that sets out to test the epidemiological (or type I) continuum model of psychosis. The authors systematically review the literature on the rates of schizophrenia symptoms surveyed in the general population. They confirmed that symptoms were more prevalent than the disorder itself; evidence of a continuum and that there were (non-specific) psychosocial factors driving this such as deprivation, drug misuse, etc. However, when they specifically tested for non-continuity within the population (i.e. are there subpopulations that are at greater risk than others?), they found strong affirmative evidence for this in studies, systematically ascertained, using sophisticated statistical methods such as coherent-cut kinetics (CCK) and factor mixture modelling (FMM). Put simply, psychopathological phenomena are continuous but risk for schizophrenia is not; there are non-arbitrary boundaries between schizophrenia and normality in the population (Linscott \& van Os, 2010).
Finally, to the moral aspects of this debate. As noted above, many commentators are drawn to the notion of a continuum because it somehow helps to counter the presumed alienating effect of making a psychiatric diagnosis. By showing that people with even the most severe forms of mental illness share much in common with the rest of us, it is hoped that we might understand our patients better and that society will stigmatize them less. These aims are naturally laudable and it may well be that proponents of the continuum will succeed in inducing these humanizing impulses generally. However, this in itself would not be justification for taking an exclusive theoretical stance on the pathogenesis or, to put it more neutrally, the underlying mechanisms for hallucinations and delusions. Indeed, it does carry a risk that by insisting that 'we are all a little bit mad...', the true seriousness and horror of major mental illnesses is trivialized and devalued, '... then no one is mad'.

\section{A way forward}

In conclusion, seeing psychotic phenomena as a point on a continuum with 'normal' experience is a useful heuristic with many positive benefits. However, like viewing a Necker cube, we can also consider the same phenomena with an eye on discontinuities and these may also provide points of theoretical growth. We should use methods and statistics that, rather than inevitably leading to continua (most psychometric methods in psychology and especially composite 'severity scales'), may also uncover potential categories. Given the likelihood that psychopathological phenomena are complex and comprise multiple dimensions, we should resist the temptation to combine them unless we have a strong theoretical justification for doing so. The null hypothesis is the continuum; we need to define in advance what would constitute evidence of discontinuity, bearing in mind error of measurement, that small populations may be lost in the tail of a normal distribution and other methodological snares. And we should use techniques when eliciting phenomena that allow us to quantify reporting bias and the demand characteristics of our questionnaires and interviews.

We need to embrace phenomenology, so that we can micro-dissect phenomena and look for specific cognitive underpinnings. By finessing the pathological hallmarks of traditional psychotic phenomena, our research risks losing the essence of psychosis. If, as some have suggested (Stip \& Letourneau, 2009), we need to look beyond old-fashioned psychotic symptoms for novel treatment targets, we need to ensure that our phenomenological skills have not atrophied 
to such an extent that we are unable to define and characterize these new entities.

Finally, we need to decide whether the focus is on distribution of traits in the population (type I) or continua of experience (type II). The former is more important for aetiological research and disease prevention whereas the latter is more relevant to phenomenology and the cognitive understanding of psychopathology.

\section{Acknowledgements}

I am grateful to Jim van Os, Tania Lincoln, Iris Sommer and Kristin Daalman for sharing data with me and for their insightful comments and to Emmanuelle Peters for sharing her expertise in this area and putting up with my silly questions.

\section{Declaration of Interest}

None.

\section{References}

Bentall RP (2003). Madness Explained: Psychosis and Human Nature. Penguin Books Ltd: London.

Charlton BG (1995). A critique of Geoffrey Rose's 'population strategy' for preventive medicine. Journal of the Royal Society of Medicine 88, 607-610.

Claridge GA (1994). A single indicator of risk for schizophrenia. Probable fact or likely myth? Schizophrenia Bulletin 20, 151-168.

Daalman K, Boks MPM, Diederen KMJ, de Weijer AD, Blom JD, Kahn RS, Sommer IEC (in press). Same or different auditory verbal hallucinations in healthy and psychotic individuals. Journal of Clinical Psychiatry.

David AS (1993). Cognitive neuropsychiatry? Psychological Medicine 23, 1-5.

David AS (1999). On the impossibility of defining delusions. Philosophy, Psychiatry, and Psychology 6, 17-20.

Everitt BS, Landau S, Leese M (eds) (2001). Cluster Analysis, 4th edn. Arnold: London.

Freeman D (2007). Suspicious minds: the psychology of persecutory delusions. Clinical Psychological Review 27, 425-457.

Garety PA, Hemsley DR (1987). Characteristics of delusional experience. European Archives of Psychiatry and Clinical Neuroscience 236, 294-298.

Horwood J, Salvi G, Thomas K, Duffy L, Gunnell D, Hollis C, Lewis G, Menezes P, Thompson A, Wolke D, Zammit S, Harrison G (2008). IQ and non-clinical psychotic symptoms in 12-year-olds: results from the ALSPAC birth cohort. British Journal of Psychiatry 193, 185-191.

Johns LC, van Os J (2001). The continuity of psychotic experiences in the general population. Clinical Psychology Review 21, 1125-1141.
Kessler RC (2002). The categorical versus dimensional assessment controversy in the sociology of mental illness. Journal of Health and Social Behavior 43, 171-188.

Kretschmer E (1925). Physique and Character, pp. 118-119. Kegan Paul, Trench, Trubner: London, UK.

Laurens KR, Hodgins S, Maughan B, Murray RM, Rutter ML, Taylor EA (2007). Community screening for psychotic-like experiences and other putative antecedents of schizophrenia in children aged 9-12 years. Schizophrenia Research 90, 130-146.

Lincoln TM (2007). Relevant dimensions of delusions: continuing the continuum versus category debate. Schizophrenia Research 93, 211-220.

Lincoln TM, Keller E (2008). Delusions and hallucinations in students compared to the general population. Psychology and Psychotherapy 81, 231-235.

Linscott RJ, van Os J (2010). Systematic reviews of categorical and continuum models of psychosis : evidence for discontinuous subpopulations underlying a psychometric continuum. Implications for DSM-V, DSM-VI and DSM-VII. Annual Review of Clinical Psychology 6, 14.1-14.29.

McGorry PD (2005). Early intervention in psychotic disorders: beyond debate to solving problems. British Journal of Psychiatry 187, s108-s110.

Meehl PE (1962). Schizotaxia, schizotypy, schizophrenia. American Psychology 17, 827-838.

Mojtabai R, Malaspina D, Susser E (2003). The concept of population prevention: application to schizophrenia. Schizophrenia Bulletin 29, 791-801.

Mullen R (2003). Delusions: the continuum versus category debate. Australia and New Zealand Journal of Psychiatry 37, 505-511.

Nayani T, David AS (1996). The auditory hallucination: a phenomenological survey. Psychological Medicine 26, 177-189.

Peters ER, Joseph SA, Day S, Garety PA (2004). Measuring delusional ideation: the 21-item Peters et al. Delusions Inventory (PDI). Schizophrenia Bulletin 30, 1005-1022.

Peters ER, Joseph SA, Garety PA (1999). Measurement of delusional ideation in the normal population: introducing the PDI (Peters et al. Delusions Inventory). Schizophrenia Bulletin 25, 553-576.

Poulton R, Caspi A, Moffitt TE, Cannon M, Murray R, Harrington HL (2000). Children's self-reported psychotic symptoms and adult schizophreniform disorder: a 15-year longitudinal study. Archives of General Psychiatry 57, 1053-1058.

Read J, Harre N (2001). The role of biological and genetic causal beliefs in the stigmatisation of 'mental patients'. Journal of Mental Health 10, 223-235.

Rose G (1992). The Strategy of Preventive Medicine. Oxford University Press: Oxford.

Simon AE, Cattapan-Ludewig K, Gruber K, Ouertani J, Zimmer A, Roth B, Isler E, Umbricht D (2009). Subclinical hallucinations in adolescent outpatients: an outcome study. Schizophrenia Research 108, 265-271.

Sommer IEC, Daalman K, Rietkerk T, Diederen KM, Bakker S, Wijkstra J, Boks MPM (2008). Healthy individuals with auditory verbal hallucinations: who 
are they? Psychiatric assessments of a selected sample of 103 subjects. Schizophrenia Bulletin. Published online: 9 October 2008. doi:10.1093/schbul/sbn130.

Stanton B, David AS (2000). First person accounts of delusions. Psychiatric Bulletin 24, 333-336.

Stip E, Letourneau G (2009). Psychotic symptoms as a continuum between normality and pathology. Canadian Journal of Psychiatry 54, 140-151.

Strauss JS (1969). Hallucinations and delusions as points on continua function. Archive of General Psychiatry 21, 581-586.

van Os J, Hanssen M, Bij1 RV, Ravelli A (2000). Strauss (1969) revisited: a psychosis continuum in the general population? Schizophrenia Research 45, 11-20.

van Os J, Linscott RJ, Myin-Germeys I, Delespaul P, Krabbendam L (2009). A systematic review and meta-analysis of the psychosis continuum: evidence for a psychosis proneness-persistence-impairment model of psychotic disorder. Psychological Medicine 39, 179-195.

Verdoux H, Maurice-Tison S, Gay B, van Os J, Salamon R, Bourgeois ML (1998). A survey of delusional ideation in primary-care patients. Psychological Medicine 28, $127-134$.

Verdoux H, van Os J (2002). Psychotic symptoms in non-clinical populations and the continuum of psychosis. Schizophrenia Research 54, 59-65.

Yung AR, Phillips LJ, Yuen HP, Francey SM, McFarlane CA, Hallgren M, McGorry PD (2003).

Psychosis prediction: 12-month follow up of a high-risk ('prodromal') group. Schizophrenia Research 60, 21-32. 